\author{
Angelo Licastro \\ (professore ordinario di Diritto ecclesiastico nell'Università degli Studi di \\ Messina, Dipartimento di Giurisprudenza)
}

\title{
Gli enti religiosi tra diritto comune e diritto speciale *
}

\author{
The Religious Bodies Between General Law and Special Legislation
}

\begin{abstract}
The present paper analyses some problematic issues concerning the legal status of "Ecclesiastical" or "Religious" Bodies. The Author underlines the growing expansion of the "not-religious" activities - such as social services, education, health care - carried out by Church Bodies and its consequences for the special features shaping an "Ecclesiastical Body".
\end{abstract}

SOMMARIO: 1. Gli elementi costitutivi della nozione di "ente ecclesiastico" e la "specialità" della relativa figura - 2. La (relativa) rigidità della componente strutturale - 3. La debole capacità connotativa della componente teleologica - 4. Il superamento del carattere marginale e controllato dello svolgimento delle attività "diverse" - 5. Su alcune criticità nei rapporti tra diritto "comune" e diritto "speciale": a) la questione dell'applicabilità agli enti ecclesiastici del decreto legislativo n. 231 del 2001 - 6. (segue) b) la questione dell'assoggettabilità degli enti ecclesiastici alle procedure concorsuali - 7. (segue) c) la questione delle divergenze risultanti dal Registro delle persone giuridiche e dal Registro delle imprese - 8. Il limitato carattere innovativo della locuzione "enti religiosi civilmente riconosciuti" presente nella normativa sul Terzo settore. Notazioni finali.

\section{1 - Gli elementi costitutivi della nozione di "ente ecclesiastico" e la "specialità" della relativa figura}

Nelle mie riflessioni conclusive - che non mirano a riepilogare in forma sintetica quanto fin qui emerso, ma a proporre alcune considerazioni personali sul tema che ci ha impegnato in questi due giorni - farò, anzitutto, riferimento alla categoria dell', ente ecclesiastico" (e, in

* Il contributo, non sottoposto a valutazione, riproduce il testo integrale, corredato delle note, della relazione presentata al Convegno su "Gli enti religiosi tra diritto speciale, diritto comune e mercati" svoltosi presso il Dipartimento di Giurisprudenza dell'Università degli Studi della Campania Luigi Vanvitelli (Caserta - Santa Maria Capua Vetere, 2-3 dicembre 2021) ed è destinato alla pubblicazione negli Atti. 
particolare, dell'ente ecclesiastico cattolico), sebbene condivida la tesi secondo la quale, stando alle direttrici della nostra Carta fondamentale, sarebbe piuttosto $\mathrm{l}^{\prime}$,"ente religioso", quale formazione sociale con fine di religione o di culto (genericamente intesa), la figura di base idonea a fondare e giustificare anche l'altra, data la priorità logica (se non anche assiologica) da riconoscere alla libertà religiosa rispetto a quella ecclesiastica ${ }^{1}$.

Nella stessa Costituzione si possono, a mio avviso, rintracciare le direttrici utili a definire gli elementi essenziali della nozione di "ente ecclesiastico" e per circoscrivere, coerentemente con essi, l'area di specialità che la caratterizza.

Sotto il primo aspetto, sappiamo che le norme di derivazione concordataria (riproposte, con qualche variante, dalla generalità delle intese con le confessioni diverse dalla cattolica) si sono orientate nel senso di considerare elementi qualificanti della figura dell'ente ecclesiastico, in forma congiunta, sia il carattere ecclesiastico che il fine di religione o di culto, entrambi presenti, ma in forma disgiunta, nella disposizione dell'art. 20 Cost. È, pertanto, ente ecclesiastico la persona giuridica derivata da una investitura della Confessione - tanto da costituire espressione, più o meno diretta, della sua organizzazione interna (carattere ecclesiastico) - che abbia, però, un fine di religione o di culto costitutivo ed essenziale, considerato immanente, nell'ordinamento giuridico statale, solo ai così detti enti "di struttura" del gruppo.

Una considerazione disgiunta di tali elementi, se può spiegarsi agevolmente nella logica sottesa a una garanzia di carattere antidiscriminatorio, come quella di cui all'art. 20 Cost. $^{2}$, non potrebbe

1 Cfr. S. BERLINGÒ, Enti e beni religiosi in Italia, il Mulino, Bologna, 1992, p. 12. Particolarmente eloquente è, in questo senso, l'art. 2 Cost., con la proclamazione, ivi operata, dell'impegno della Repubblica alla salvaguardia dei "diritti inviolabili dell'uomo" anche nelle formazioni sociali dove si svolge la sua personalità, da cui deriva che prima e alla base di ogni forma di tutela dell'autonomia delle formazioni sociali, nella nostra Carta costituzionale, sta il fondamentale principio personalistico, che sorregge e connota le molteplici forme di libertà e di autonomia dalla medesima garantite. Scarsa o nessuna rilevanza ha, sotto questo profilo, la circostanza che la dimensione più antica della libertà di religione è la libertas Ecclesiae, ossia la "libertà della istituzione ecclesiastica e della comunità dei fedeli di fronte al potere politico": cfr. S. FERRARI, La libertà di religione è un diritto universale?, in G. D'ANGELO, G. FAUCEGLIA (a cura di), Rigore e curiosità. Scritti in memoria di Maria Cristina Folliero, t. I, a cura di G. D'ANGELO, Torino, Giappichelli, 2018, p. 415.

2 ... in base alla quale "la circostanza di un radicamento confessionale non può di per sé comportare un trattamento peggiore o migliore rispetto agli enti che, pur avendo la 
essere coerentemente invocata in presenza di un collegamento strutturale che fa dell'ente una cellula organizzativa espressione dell'identità del gruppo. Questo perché l'ecclesiasticità dell'ente riflette i vantaggi e i limiti connessi con le garanzie costituzionali di cui è circondato il principio di autonomia confessionale (art. 7, primo comma, e art. 8, secondo comma, Cost.) $)^{3}$ : questo principio assicura la non ingerenza dello Stato nella vita e nell'organizzazione interna della confessione - di cui gli enti ecclesiastici sono, come si diceva, espressione e tramite - entro, tuttavia, l'ambito operativo riferibile all' ",ordine" religioso, che contraddistingue e qualifica lo stesso gruppo come "confessione". Quando la normativa di derivazione concordataria richiede di compiere una verifica (ad esempio, in sede di riconoscimento della personalità giuridica) sull'effettivo perseguimento del fine di religione o di culto è come se volesse cautelarsi dal rischio che le garanzie costituzionali particolarmente rigide di autonomia organizzativa delle confessioni siano indebitamente utilizzate per ambiti

medesima finalità religiosa, non sono però collegati ad una vera e propria organizzazione": S. BERLINGÒ, Enti e beni religiosi in Italia, cit., p. 65.

3 Ha sottolineato opportunamente come l'incidenza sulla disciplina dell'ente ecclesiastico civilmente riconosciuto delle norme derivanti dall'ordinamento confessionale di appartenenza - accanto alle "norme statuali a carattere generale" e a quelle "a carattere speciale" - si spieghi "in ragione della peculiare rilevanza riconosciuta alle autonomie confessionali rispetto alle altre autonomie sociali", P. PICOZZA, Note a margine di un caso di «ecclesiasticizzazione» di società commerciale, in Quad. dir. pol. eccl., 199192/1, p. 555. Cfr., altresì, P. CONSORTI, L'impatto del nuovo Codice del Terzo settore sulla disciplina degli "enti religiosi", in Stato, Chiese e pluralismo confessionale, Rivista telematica (https://www.statoechiese.it), n. 4 del 2018, p. 5, secondo cui "la Costituzione riserva un'area di specialità agli enti ecclesiastici (confessionali) per il solo fatto di essere espressione dell'autonomia confessionale", nonché P. FLORIS, Autonomia confessionale. Principi-limite fondamentali e ordine pubblico, Jovene, Napoli, 1992, p. 177 ss. Analogamente, G. D'ANGELO, Declinazioni giuridiche del fine di religione e di culto. Dalla forma all'interesse, Giappichelli, Torino, 2020, p. 78, secondo cui la figura dell'ente ecclesiastico civilmente riconosciuto "[a]ttiene in larga parte proprio a un essenziale profilo di autonomia confessionale", e P. CAVANA, Gli enti ecclesiastici nel sistema pattizio, $2^{\mathrm{a}}$ ed., Giappichelli, Torino, 2011, p. 128 e p. 145 ss. Sottolinea il collegamento tra autonomia della confessione e specialità del regime giuridico dell'ente ecclesiastico anche G. CASUSCELLI, Enti ecclesiastici, in Commentario del codice civile diretto da E. GABRIELLI, Delle persone, a cura di A. BARBA e S. PAGLIANTINI, Utet, Torino, 2013, p. 372, secondo cui la specialità della disciplina dell'ente ecclesiastico non può "operare per impedire il naturale espandersi di un diritto comune (vuoi che imponga obblighi o oneri, vuoi che riconosca situazioni giuridiche di vantaggio) inidoneo a incidere quell'autonomia". 
operativi estranei a quello distintivo e caratterizzante l'identità del gruppo ${ }^{4}$

Naturalmente questo vale solo in linea di massima, o in via tendenziale, in quanto la confessione (per il tramite dei suoi enti) ha modo di proiettarsi, nel suo agire, anche nella sfera di rilevanza delle attività meramente civili o profane, in relazione alla quale sovrano è però lo Stato e la peculiare identità del gruppo può ridursi anche solo nei limiti del riconoscimento di una particolare tendenza. È vero che esiste un divieto di trattamento discriminatorio (almeno a prima vista) inclusivo di "ogni forma di attività dell'ente" (art. 20 Cost.), ma esso non può significare estensione automatica, totale e incondizionata del trattamento comparabilmente più vantaggioso, con la garanzia del mantenimento inalterato di tutte le caratteristiche identitarie $e$ di specialità dell'ente ecclesiastico 5 .

Comunque sia, per il momento basta osservare, pure quanto alla circoscrizione dell'area di specialità della disciplina dell'ente ecclesiastico ${ }^{6}$, che al cuore di tale disciplina - condizione per potere affermare la stessa "perdurante validità della figura"7 - sta il nesso con lo specifico ordinamento della confessione e il perseguimento del fine di religione o di culto.

\footnotetext{
${ }^{4}$ Di recente, si è ribadito a ragione che il fine di religione e di culto "ripropone [...] una dialettica di fondo che, al di là delle sue differenti vesti storico-evolutive, si riporta al nucleo essenziale del diritto ecclesiastico e ne spiega l'inquietudine costitutiva": G. D'ANGELO, Declinazioni giuridiche, cit., p. 17. Secondo lo stesso A., il fine di religione e di culto "risponde prevalentemente all'esigenza di qualificare soggettivamente un dato ente e circoscrivere l'ambito di rilevanza del suo vincolo confessionale" (ivi, p. 119, il primo corsivo è presente nell'originale, il secondo è mio).

${ }^{5}$ Cfr. G. CASUSCELLI, Enti ecclesiastici, cit., p. 341, secondo cui la "specialità della disciplina non può essere contrassegnata [...] dalla coesistenza contraddittoria del modello operativo dell'inclusione/esclusione governato dalla regola del maggior vantaggio dell'ente ecclesiastico".

"Si tratta di un "diritto speciale" a tutti gli effetti, non solo perché contenuto in fonti di produzione "specializzate" (quali sono le fonti concordatarie per gli enti della Chiesa cattolica o quelle frutto di intesa per gli enti delle altre confessioni), ma anche perché caratterizzato da regole speciali, cioè per diversi aspetti diverse da quelle applicabili agli enti di diritto comune.

${ }^{7} \mathrm{Si}$ è osservato che "[a]sserire la perdurante validità della figura dell'ente ecclesiastico civilmente riconosciuto", sia pure "ridisegnata nella sostanza e nell'ambito di estensione", "in tanto ha [...] un senso, in quanto si affermi necessariamente anche l'esistenza di un regime giuridico tipico del genere di persona giuridica così individuato": P. PICOZZA, Note a margine, cit., p. 553 s.; ID., L'ente ecclesiastico civilmente riconosciuto, Giuffrè, Milano, 1992, p. 189 ss.
} 
Dall'uno e dall'altro elemento bisogna partire per valutare entro quali limiti abbia senso, e sia costituzionalmente praticabile, un trattamento "speciale" dell'ente.

\section{2 - La (relativa) rigidità della componente strutturale}

Conseguenza della prima caratteristica (nesso con lo specifico ordinamento della confessione) è la speciale conformazione strutturale dell'ente, molto bene messa in luce dall'Intesa interpretativa tra Italia e Santa Sede del 19978.

Com'è noto, in sede di riconoscimento della personalità giuridica degli enti ecclesiastici, la prassi amministrativa, confortata da un preciso orientamento del Consiglio di Stato 9 , si era orientata nel senso di procedere a una serie di verifiche su presupposti, anche di forma, richiesti dal codice civile per l'attribuzione della personalità agli enti privati. Tutto ciò indusse la Conferenza episcopale italiana a manifestare una serie di riserve verso quella che poteva comprensibilmente apparire come una tendenza (ancora più marcata di quella risultante dalle stesse norme pattizie) ${ }^{10}$ alla riduzione al diritto comune del regime giuridico degli enti ecclesiastici. Non a caso si è potuto affermare che il testo poi concordato, nell'affrontare i singoli profili controversi, accogliendo buona parte delle richieste di provenienza confessionale, ruoterà "attorno ad un'unica

8 Scambio di Note con Allegati 1 e 2 tra la Repubblica Italiana e la Santa Sede costituente un'intesa tecnica interpretativa ed esecutiva dell'Accordo modificativo del Concordato Lateranense del 18 febbraio 1984 e del successivo Protocollo del 15 novembre 1984, Vaticano-Roma, 10 aprile/30 aprile 1997, in Suppl. ord. Gazz. Uff. 15 ottobre 1997, n. 241, riprodotto per estratto in S. BERLINGÒ, G. CASUSCELLI, Codice del diritto ecclesiastico, $5^{\mathrm{a}}$ ed., con la collaborazione di A. LICASTRO e M. TOSCANO, Giuffrè, Milano, 2009, p. 601 ss.

9 Cfr., ad esempio, Cons. St., parere 31 maggio 1995, n. 1607, in Quad. dir. pol. eccl., 1996/3, p. 965 s.; Id., parere 2 dicembre 1992, n. 3012, ivi, 1993/3, p. 975; Id., 27 novembre 1991, n. 2717, ivi, 1991-92/1, p. 546; Id., parere 23 ottobre 1991, n. 2453, ivi; Id., parere 24 settembre 1991, n. 2102, ivi, p. 541 s.; Id., parere 24 aprile 1991, n. 957, ivi, p. 541; Id., parere 5 luglio 1990, n. 952, ivi, 1990/1, p. 745 s.; Id., parere 6 giugno 1990, n. 2031/88, ivi, p. 745; Id., parere 23 maggio 1990, n. 640, ivi, p. 744 s.; Id., parere 10 gennaio 1990, n. 2434/89, ivi, p. 743; Id., parere 28 giugno 1989, n. 1174/89, ivi, p. 741; Id., sez. I, parere 10 maggio 1989, n. 767, ivi, p. 740.

10 Sulla sempre più estesa riconduzione nell'ambito del diritto privato del regime giuridico degli enti ecclesiastici, si veda, per tutti, A. FUCCILLO, Le nuove frontiere dell'ecclesiasticità degli enti. Struttura e funzione delle associazioni ecclesiastiche, Jovene, Napoli, 1999. Individua nel d.P.R. n. 361 del 2000 alcuni indici di una tendenza inversa, P. CAVANA, Gli enti ecclesiastici, cit., p. 212. 
essenziale questione", quella della "specialità degli enti ecclesiastici in opposizione alla loro eventuale assoggettabilità al diritto comune"11.

Il suddetto modo di procedere dell' Amministrazione portava, nella sostanza, a una sorta di "rifondazione" dell'ente ecclesiastico nell'ordinamento italiano, in contrasto con l'impegno, pattiziamente assunto dalla Repubblica, "ad accogliere nel proprio ordinamento gli enti ecclesiastici, ai quali accorda il riconoscimento, con le caratteristiche che agli stessi ineriscono nell'ordinamento di provenienza"12. L'irriducibile specialità degli enti ecclesiastici è, appunto, quella che essi "sono riconosciuti come persone giuridiche agli effetti civili nel rispetto delle loro caratteristiche originarie stabilite dalle norme del diritto canonico"13.

Il collegamento genetico con l'ordinamento confessionale ha poi modo di proiettarsi nell'ordinamento civile anche durante tutta la vita dell'ente e lo svolgimento delle sue attività.

Il nesso è così stretto che sia le limitazioni dei poteri degli organi di rappresentanza dell'ente, autonomamente fissate dal gruppo, sia i controlli canonici sull'amministrazione dei beni, sono civilmente rilevanti, una volta adempiuto l'onere della registrazione e nei termini di quanto previsto dall'art. 18 della legge n. 222 del 1985.

$\mathrm{Su}$ questi aspetti essenziali della componente strutturale dell'ente ecclesiastico, in grado di esprimere il tratto più marcato e caratteristico di specialità della disciplina a esso relativa ${ }^{14}$, è difficile che possano incidere radicalmente gli sviluppi della normativa di diritto comune: la normativa di derivazione pattizia - in un ordinamento come il nostro, incline, per scelta costituzionale, alla cooperazione con le confessioni religiose - è da considerare, piuttosto, sede ideale, se non tramite essenziale, per il collegamento tra i peculiari assetti organizzativi interni della Chiesa e le loro ricadute nella sfera dell'ordinamento civile. Si potrà registrare qualche attrito tra alcuni sviluppi del diritto comune e circoscritti profili concernenti la libertà organizzativa della confessione, ma gli accennati

11 C. CARDIA, Alcune innovazioni in materia di enti ecclesiastici, in Quad. dir. pol. eccl., $1997 / 3$, p. 917.

12 Scambio di Note, cit., Relazione (mio il corsivo). L'impegno pattizio risulterebbe dall'art. 7, n. 2, dell'Accordo tra Stato e Chiesa del 18 febbraio 1984, nella parte in cui è previsto che siano riconosciuti agli effetti civili gli enti "eretti o approvati secondo le norme del diritto canonico".

13 Scambio di Note, cit., Documento conclusivo (mio il corsivo).

${ }^{14}$ Ricordo che l'intesa interpretativa del 1997 precisa che non sono applicabili agli enti ecclesiastici "le norme dettate dal codice civile in tema di costituzione, struttura, amministrazione ed estinzione delle persone giuridiche private". 
tratti di specialità dell'assetto strutturale dell'ente ecclesiastico sembrano definiti in maniera piuttosto rigida e non rischiano di essere stravolti o di essere messi seriamente in discussione.

È anche per questo difficile che il ricorso agli strumenti offerti dall'autonomia privata possa garantire risultati del tutto equivalenti a quelli garantiti dalla disciplina pattizia.

Si è, ad esempio, da tempo concordi nel ritenere che un ente ecclesiastico possa dare vita o concorrere a dare vita a una società commerciale (oltre che acquistare partecipazioni in società già costituite) ${ }^{15}$ : pur con tutti gli sforzi volti a conservare - attraverso le previsioni dello statuto - un qualche specifico nesso con la confessione, fino a "ecclesiasticizzarne" la struttura16 (nei limiti consentiti dal principio di autonomia dell'ente rispetto all'influenza di soggetti esterni), la società non sarà mai, però, un ente ecclesiastico, ma solo una sua emanazione, che opera come soggetto autonomo, restando, a mio parere, interamente sottoposto al diritto comune.

Senza trascurare che in presenza di questo tipo di clausole statutarie volte a disciplinare il collegamento con la confessione, si potrebbero configurare anche rischiose forme di responsabilità da "direzione e coordinamento di società" in capo alla Chiesa, come nel caso in cui l'ente ecclesiastico controllasse una impresa sociale costituita sotto forma di società (art. 2497 ss. c.c., richiamati dall'art. 4, secondo comma, del decreto legislativo n. 112 del 2017) ${ }^{17}$.

15 Per tutti si veda A. FUCCILLO, Diritto religioni culture. Il fattore religioso nell'esperienza giuridica, $3^{\mathrm{a}}$ ed., Giappichelli, Torino, 2019, p. 151 ss.

16 Per l'uso di questa espressione, riferita ad alcune previsioni statutarie di una società finalizzate a condizionare determinati atti gestori a decisioni di soggetti confessionali esterni all'organizzazione dell'ente, cfr. P. PICOZZA, Note a margine, cit., p. 552.

17 Prospetta questo tipo di rischio, P. CAVANA, Gli enti ecclesiastici nel processo di riforma del Terzo settore. Profili ricostruttivi e applicazioni pratiche, in ID. (a cura di), Gli enti ecclesiastici nella riforma del Terzo settore, Giappichelli, Torino, 2021, p. 58, il quale ricorda, altresì, che "qualsiasi forma di collegamento tra un ente ecclesiastico civilmente riconosciuto ed una società commerciale, attuata per via statutaria o in altra forma e riconducibile allo schema indicato, è destinata a ricadere sotto questa normativa" (p. 59). Cfr. pure A. PERRONE, V. MARANO, La riforma del Terzo settore e gli enti ecclesiastici: un rischio, un costo o un'opportunità?, in Stato, Chiese e pluralismo confessionale, cit., n. 35 del 2018, p. 10 s., nonché G. CASUSCELLI, Enti ecclesiastici, cit., p. 431, che, sia pure in una prospettiva di riforma della disciplina vigente riguardante gli enti ecclesiastici, sottolinea l'analogia tra la "posizione di controllo e di direzione" rivestita dalla confessione rispetto ai propri enti esponenziali e lo schema di cui all'art. 2497 c.c. 


\section{3 - La debole capacità connotativa della componente teleologica}

$\grave{E}$, invece, la componente teleologica, o, se si preferisce, il requisito finalistico, ad avere rivelato, col passare del tempo, livelli di flessibilità o margini di ambiguità o di ambivalenza tali da non riuscire più a porsi, nel concreto agire e operare dell'ente in ambito civile, come indice effettivamente distintivo della figura in esame e capace di circoscriverne, in modo rigoroso, la relativa specialità. Tanto da dovere assistere, come è stato osservato, a un "declino del carattere non lucrativo del "fine di culto e di religione"», a un suo "progressivo attenuarsi" e all' «emergente rilievo del "fine di profitto indiretto"» 18.

Elementi di ambiguità sono, a questo riguardo, presenti nella stessa normativa pattizia.

Intanto, per un numero consistente di enti (di nuova costituzione) ${ }^{19}$, il perseguimento del fine di religione o di culto è oggetto di presunzione assoluta (art. 2, primo comma, 1. n. 222 del 1985), con riflessi, in questi casi, in ordine alla praticabilità stessa - già non agevole in via generale dell'istituto della revoca del riconoscimento ${ }^{20}$; non è poi mai stato

18 Sono tutte espressioni di G. CASUSCELLI, Enti ecclesiastici e doveri di solidarietà, in Stato, Chiese e pluralismo confessionale, cit., n. 7 del 2014, p. 7. Cfr. pure G. D'ANGELO, Declinazioni giuridiche, cit., p. 137 s.

19 Invita opportunamente a tenere conto degli effetti prodotti dalla "conservazione della personalità giuridica degli enti preesistenti", spesso " "ecclesiastici" per il solo fatto del collegamento con una confessione, talvolta tali solo per antico possesso di stato", G. CASUSCELLI, Enti ecclesiastici, cit., p. 394.

${ }^{20}$ Cfr. S. BERLINGÒ, Enti ecclesiastici - Enti delle Confessioni religiose, in Stato, Chiese e pluralismo confessionale, cit., maggio 2007, p. 10 s., che esprime dubbi circa la "plausibilità ed efficienza del ricorso a questo meccanismo" nei casi richiamati. Sembra, invece, profilare (in occasione del riconoscimento, ma con considerazioni estensibili anche al momento della revoca) l'ammissibilità di una verifica volta ad accertare la corrispondenza alla figura tipica cui fa riferimento la denominazione, Cons. St., sez. I, parere 12 maggio 1993, n. 462, in Quad. dir. pol. eccl., 1994/3, p. 924 s. Sul punto cfr. anche C. CARDIA, Manuale di diritto ecclesiastico, il Mulino, Bologna, 1996, p. 346 s.

$\mathrm{Su}$ di un piano generale, non mancano i tentativi volti a individuare strumenti alternativi alla revoca del riconoscimento da far valere nei confronti dell'ente ecclesiastico che perda la sua primaria (e primigenia) connotazione finalistica: si è richiamato alla possibile disapplicazione del decreto di riconoscimento P. LO IACONO, Gli enti ecclesiastici e gli enti religiosi quale archetipo della personalità giuridica (La valenza funzionale della personificazione tra normativa pattizia e Codice del Terzo settore), in P. CAVANA (a cura di), Gli enti ecclesiastici, cit., p. 113 ss. e si è pure sostenuto che la stessa normativa sul terzo settore, se applicata all'ente ecclesiastico, possa offrire risorse utilizzabili a questo fine: cfr. P. FLORIS, Enti religiosi e riforma del Terzo settore: verso nuove partizioni nella 
definitivamente acclarato il dubbio che l'ente ecclesiastico possa non solo svolgere attività diverse da quelle di religione o di culto, ma perseguire anche finalità diverse e del tutto eterogenee rispetto a quella istituzionale (dando la legge indicazioni precise solo per le finalità di carattere caritativo previste dal diritto canonico, da considerare connesse alla finalità di religione o di culto, di cui anzi concorrono a definire il carattere costitutivo ed essenziale) ${ }^{21}$; si è, poi, concordi nel ribadire che le attività diverse non possono assumere carattere soverchiante, fino al punto da risultare prevalenti, ma - a parte l'assenza di momenti di verifica del permanere della coerenza tra le attività svolte dall'ente e la sua finalità istituzionale - non si offrono elementi alla luce dei quali definire concretamente tale situazione e affermarne (o escluderne) la ricorrenza.

In particolare, per quanto riguarda quest'ultimo punto, pure aderendo alla tesi che l'ente ecclesiastico sia titolato a perseguire finalità diverse da quella istituzionale e che, quindi, non sorga una questione di necessaria strumentalità, rispetto alla finalità di culto, delle attività diverse, non è detto che ci si debba rifare, per accertare la loro natura secondaria o non prevalente, a un dato meramente quantitativo, che prescinda del tutto dalla qualità oggettiva delle predette attività; o che la verifica di carattere quantitativo debba essere fatta in termini aritmetici guardando ai dati contabili, ad esempio, sui ricavi in rapporto alle entrate o ai costi complessivi, alla stessa stregua del parametro adottato dal legislatore nel definire i criteri e i limiti ai fini dell'esercizio delle attività diverse da parte degli enti del Terzo settore in rapporto a quelle di interesse generale da svolgere in via esclusiva o principale ${ }^{22}$.

Se appare, dunque, fondata l'impressione che tutti questi fattori di oggettiva incertezza abbiano contribuito a "depotenziare", per così dire, la

disciplina degli enti religiosi, in Stato, Chiese e pluralismo confessionale, cit., n. 3 del 2018, p. 18 s.

${ }^{21}$ Il dato normativo di cui all'art. 2, terzo comma, della legge n. 222 del 1985, che prevede la connessione del fine di religione o di culto (soltanto) con "finalità di carattere caritativo previste dal diritto canonico", non pare possa essere, invece, utilizzato a favore della tesi prevalente, favorevole ad ammettere la pluralità di fini anche del tutto eterogenei rispetto a quello di culto. Cfr. C. CARDIA, Manuale, cit., p. 347 ss. Il perseguimento di una pluralità di fini caratterizza, invece, gli enti disciplinati dalle intese con le confessioni diverse dalla cattolica.

${ }^{22}$ Cfr. il Decreto del Ministero del lavoro e delle politiche sociali del 19 maggio 2021, n. 107 [Regolamento ai sensi dell'articolo 6 del decreto legislativo n. 117 del 2017 (Codice del Terzo settore), concernente l'individuazione di criteri e limiti delle attività diverse], in Gazz. Uff. 2 agosto 2017, n. 179. Esprime motivate riserve sull'adeguatezza di "indici meramente economici", P. CONSORTI, L'impatto del nuovo Codice, cit., p. 18. 
centralità dell'elemento teleologico intorno al quale la normativa pattizia aveva costruito la figura soggettiva in esame, forti suggestioni nella medesima direzione provengono poi dalla legislazione di diritto comune.

In questo caso, l'immagine del fine di religione o di culto "debolmente connotativo" di una tipologia speciale di ente, dipende dagli sviluppi più recenti della legislazione in tema di soggettività giuridica ${ }^{23} \mathrm{e}$ di condizione giuridica degli enti senza scopo di lucro ${ }^{24}$, per i quali ultimi, dalla posizione, di ascendenza ottocentesca, caratterizzata da sostanziale diffidenza, si è passati a una volta addirittura a incentivarne la costituzione e l'azione, almeno a condizione che possa trarne beneficio la soddisfazione di interessi generali di cui lo Stato si fa garante e promotore.

La premessa, spesso non esplicitata, ma talvolta sottesa a un tale ragionamento, è che la specialità implichi necessariamente la deroga mirata selettivamente a favorire (magari dal punto di vista fiscale) qualcuno o qualcosa, consenta di ritagliarsi spazi di azione preclusi agli altri, si traduca, in definitiva, in disciplina di carattere privilegiario ${ }^{25}$.

Ma incidono anche altri fattori, su cui si tornerà a breve.

\section{4 - Il superamento del carattere marginale e controllato dello svolgimento delle attività "diverse"}

Senza dubbio, la disposizione da cui emerge in maniera maggiormente esplicita l'esigenza di contemperare o di raccordare la condizione giuridica speciale dell'ente ecclesiastico con la normativa di diritto comune è quella contenuta nel secondo inciso dell'art. 7, n. 3, dell'Accordo 18 febbraio 1984 tra Stato e Chiesa cattolica, secondo cui, com'è noto, "[1]e attività diverse da quelle di religione o di culto, svolte dagli enti

${ }^{23} \mathrm{Ne}$ rimarcano, fra gli altri, l'importanza, G. CASUSCELLI, Enti ecclesiastici, cit., p. 319 s., e M. BASILE, voce Enti non lucrativi (diritto civile), in Enc. dir., Aggiornamento, III, Giuffrè, Milano, 1999, p. 580.

24 Reputa, invece, che l'elemento finalistico si mantenga centrale nella disciplina dell'ente ecclesiastico, anche dopo il Codice del terzo settore, P. LO IACONO, Gli enti ecclesiastici, cit., p. 86.

${ }^{25}$ Ricorda, invece, opportunamente G. CASUSCELLI, Enti ecclesiastici, cit., p. 338, che gli enti ecclesiastici «meritano (o necessitano di) una disciplina specifica (e talvolta) speciale - segnata anche da un approccio promozionale - non per la qualificazione e l'appartenenza che li connota, o per la loro "natura" (elementi che, di per sé non potrebbero conferire uno status privilegiato), ma perché e in quanto strumenti per la concreta realizzazione di fini-valori e principi costituzionali». 
ecclesiastici, sono soggette, nel rispetto della struttura e della finalità di tali enti, alle leggi dello Stato concernenti tali attività e al regime tributario previsto per le medesime".

Questa disposizione sempre più tende a funzionare come "porta d'accesso" per legittimare iniziative gestionali e operative lontane dal tipico "paradigma funzionale" e "strutturale" dell'ente ${ }^{26}$. Col risultato che la relativa figura soggettiva può apparire ormai come "un (fragile) contenitore di realtà diverse, talora lontane dall'essere caratterizzate da finalità di culto e di religione con carattere costitutivo ed essenziale" 27 . L'estensione dell'applicazione del diritto comune, per effetto della crescente espansione delle attività "diverse"28, secondo alcune letture, avrebbe ormai finito con lo stravolgere il volto stesso dell'ente ecclesiastico, facendogli assumere ordinariamente i tipici connotati dell'ente commerciale. A fare da pendant di altre controverse ibridazioni, se non di vere e proprie metamorfosi già da tempo segnalate, e più sopra ricordate ${ }^{29}$, bisognerebbe, dunque, prendere atto della tendenza ormai diffusa alla "commercializzazione" della figura dell'ente ecclesiastico ${ }^{30}$.

26 G. CASUSCELLI, Enti ecclesiastici e doveri di solidarietà, cit., p. 8, che invita a considerare come causa del superamento del paradigma funzionale non solo lo svolgimento di attività diverse, ma anche "l'implementazione e/o trasformazione delle attività proprie, ma secondarie (basti pensare alle vicende degli oratori parrocchiali)" (sottolineatura presente nell'originale).

27 G. CASUSCELLI, Enti ecclesiastici e doveri di solidarietà, cit., p. 8 s. Parla di "concetto duttile e poliforme in quanto tale idoneo a fungere da copertura giuridica a diverse esigenze pratico/applicative che poco o nulla hanno a che fare con la sua archetipica funzione", M. FERRANTE, Enti religiosi/ecclesiastici e riforma del terzo settore, $2^{\mathrm{a}}$ ed., Giappichelli, Torino, 2019, p. 62. Stessa sorte, del resto, era toccata agli enti disciplinati dal libro I del codice civile, trasformatisi in "una sorta di scatolone vuoto che il legislatore, di volta in volta, riempie surrettiziamente di contenuti ritenuti più opportuni per il raggiungimento dei fini (spesso politici) che si propone di perseguire": ivi, p. 3.

28 Come molti altri, parla di "crescita del ruolo delle confessioni nello svolgimento di attività diverse da quelle di religione o di culto", I. BOLGIANI, Gli effetti della riforma del terzo settore in materia di "enti religiosi civilmente riconosciuti». Normativa, buone prassi ed esigenze del tessuto sociale, Giappichelli, Torino, 2021, p. 17.

${ }^{29}$ Cfr. P. PICOZZA, Note a margine, cit., p. 552 ss.

${ }^{30}$ Cfr. P. CAVANA, Gli enti ecclesiastici nel processo di riforma, cit., p. 20 s. Si veda, pure, A. BETTETINI, Le attività dell'ente ecclesiastico a confronto con la normativa civile. Profili problematici, in L'ente ecclesiastico a trent'anni dalla revisione del Concordato, a cura di P. CLEMENTI e L. SimONELli, Giuffrè, Milano, 2015, p. 224 ss.; M. FERRANTE, Enti religiosi/ecclesiastici, cit., p. 62 s., che parla di «esasperata tensione dialettica tra ecclesiasticità e commercialità degli enti che ha finito con l'alterare il rapporto tra "struttura e funzione" degli enti ecclesiastici». 
A un esito di questo tipo si sarebbe giunti, anzitutto, a causa del travolgente effetto prodotto dall'abolizione dell'autorizzazione agli acquisti (art. 17 c.c.) ${ }^{31}$, che in passato era servita ad assicurare una costante verifica di coerenza tra beni acquistati dall'ente e svolgimento della finalità istituzionale (o di attività a essa in qualche modo strumentali) ${ }^{32}$. Nei fatti, l'istituto relegava a una condizione di sostanziale "marginalità" lo svolgimento delle attività diverse, con l'effetto indiretto di esaltare $i$ tratti identitari più caratteristici dell'ente ecclesiastico ${ }^{33}$.

Ma contribuisce (a quell'esito) forse pure la tendenza a leggere la clausola del rispetto della struttura e della finalità - posta a presidio dell'identità dell'ente ecclesiastico, almeno secondo l'interpretazione maggiormente aderente alla lettera della disposizione ${ }^{34}$ - come capace di assicurare all'ente stesso deroghe rispetto alla legislazione di diritto comune, senza che abbia trovato grosso seguito la tesi favorevole a riconoscerle piuttosto l'effetto di un possibile condizionamento del legittimo svolgersi delle attività diverse, in presenza di una situazione di chiara incompatibilità di queste con la struttura o la finalità dell'ente ${ }^{35}$.

${ }^{31}$ L'abolizione è stata operata dall'art. 13 della legge 15 maggio 1997, n. 127.

32 Cfr. S. BERLINGÒ, L'autorizzazione agli acquisti degli enti ecclesiastici tra autonomia, vigilanza e controllo: dai Patti lateranensi agli accordi di Villa Madama, in AA. VV., L'autorizzazione agli acquisti degli enti ecclesiastici e degli altri enti senza fine di lucro, in Jus, 1993, p. 271; C. CARDIA, Alcune innovazioni, cit., p. 932, il quale aveva espresso il timore che l'abolizione dell'istituto avrebbe finito col "lambire un principio cardine della disciplina di tali enti, per il quale essi in tanto sono riconosciuti e tutelati con qualche consistente privilegio in quanto nascono e agiscono continuativamente nell'ordinamento civile con quelle caratteristiche genetiche di ecclesiasticità (finalità - struttura - attività) che ne determinano la specialità" (corsivo presente nell'originale).

${ }^{33}$ Cfr. P. CAVANA, Gli enti ecclesiastici nel processo di riforma, cit., p. 19.

${ }^{34}$ La clausola, ancora una volta, esalta i profili soggettivi o speciali dell'ente, espressivi della sua identità, rispetto a quelli oggettivi concernenti le attività: P. CAVANA, Gli enti ecclesiastici, cit., p. 190.

35 Tale seconda interpretazione non è priva di puntuale riscontro in giurisprudenza: cfr. Cons. St., sez. I, parere 6 giugno 1990, n. 758, in Quad. dir. pol. eccl., 1991-92/1, p. 534 s., secondo cui le attività diverse da quelle di religione e di culto (nel caso di specie, attività di conduzione di un ospedale) "sono consentite purché compatibili con la struttura e la finalità dell'ente". Essa potrebbe, inoltre, risultare in qualche modo indirettamente confermata dall'art. 15 della legge n. 222 del 1985, dove si precisa che " $[\mathrm{g}] \mathrm{li}$ enti ecclesiastici civilmente riconosciuti possono svolgere attività diverse da quelle di religione o di culto, alle condizioni previste dall'articolo 7, n. 3, secondo comma, dell'accordo del 18 febbraio 1984" (mio, naturalmente, il corsivo). Secondo C. CARDIA, Manuale, cit., p. 379, "non c'è dubbio che un orizzonte di compatibilità esiste, nel senso che non è pensabile che un ente ecclesiastico chieda e ottenga il riconoscimento per poi gestire o una finanziaria, o una catena di hotel di lusso, o attività similari" (corsivo 
Una ulteriore spinta all'attrazione dell'ente ecclesiastico verso "modelli operativi e funzionali tipici dell'ente commerciale", se non addirittura verso processi di "secolarizzazione" vera e propria della figura ${ }^{36}$, potrebbe provenire, secondo alcuni Autori, dalla stessa riforma del Terzo settore, dato il suo carattere incentivante dello svolgimento di attività di interesse generale, da inquadrare ancora una volta, dalla prospettiva della legge n. 222 del 1985, nell'ambito delle attività "diverse". La stessa sensibilità manifestata dal legislatore verso il rispetto della struttura e della finalità dell'ente, nel momento in cui si tratta di recepire nel regolamento del ramo le disposizioni del Codice, è stata letta come un fattore che favorisce questo tipo di trasformazione ${ }^{37}$.

Bisogna, però, pure segnalare, su di un piano tecnico-formale, che lo svolgimento delle attività di interesse generale assume necessariamente carattere principale o prevalente nel caso dell'ente del Terzo settore tout court, mentre esse, anche se svolte in forma imprenditoriale, nel caso dell'ente ecclesiastico, sono pur sempre attività principali o prevalenti nell'ambito del ramo di attività di Terzo settore. Su di un piano poi più sostanziale e concreto, dovrebbe essere la confessione in grado di valutare sapientemente, e con bilanciamento tra tutti i costi e benefici, se cogliere le opportunità che vengono offerte dalla nuova normativa o se, invece, considerarla di volta in volta non adeguata anche e soprattutto in rapporto alle peculiari esigenze identitarie del gruppo ${ }^{38}$. Il recepimento, infine, della clausola del rispetto della struttura e della finalità in una fonte di diritto comune, qualunque sia in concreto il suo valore "incentivante" delle attività "diverse", è di per sé una semplice attuazione della corrispondente previsione contenuta nella fonte di derivazione bilaterale.

presente nell'originale). L'A. interpreta, peraltro, la clausola in discorso come garanzia di non ingerenza per il nucleo confessionale dell'ente in caso di controlli o ispezioni (ivi). 22.

36 P. CAVANA, Gli enti ecclesiastici nel processo di riforma, cit., rispettivamente p. 21 e p.

37 P. CAVANA, Gli enti ecclesiastici nel processo di riforma, cit., p. $25 \mathrm{~s}$.

38 Ricordo che l'art. 14, terzo comma, del D.M. 106 del 2020 ha previsto, tra l'altro, che alla domanda di iscrizione al RUNTS dell'ente religioso civilmente riconosciuto deve essere allegato l'atto con il quale la competente autorità religiosa autorizza l'iscrizione o dichiara che tale autorizzazione non è necessaria. Ritiene che ci si debba domandare se l'adesione «al regime delineato dalla riforma, non rischi di "distrarre" gli enti in questione dal proprio campo di azione, "barattando" il fine di religione o di culto con quello di solidarietà sociale», I. BOLGIANI, Gli effetti della riforma, cit., p. 135. 


\section{5 - Su alcune criticità nei rapporti tra diritto "comune" e diritto "speciale": a) la questione dell'applicabilità agli enti ecclesiastici del decreto legislativo $n .231$ del 2001}

Vero è che si è di fronte a una ridefinizione degli equilibri tra normativa speciale e normativa di diritto comune che può essere causa di confusioni o contraddizioni e può porre problemi di coerenza all'interno dell'ordinamento civile, atteso il carattere promozionale, in molti ambiti, della legislazione riguardante gli enti ecclesiastici.

Proporrò ora alcuni esempi di criticità nei rapporti tra diritto comune e diritto speciale, senza alcuna pretesa di avere individuato i più significativi.

La regola generale della sottoposizione al diritto comune delle attività diverse dovrebbe comportare l'applicabilità all'ente ecclesiastico della normativa riguardante la responsabilità degli enti per gli illeciti amministrativi dipendenti da reato, mancando, nella relativa disciplina, una norma esonerativa. La questione, in vero, è discussa in dottrina, ma, sia pure con vari distinguo, sembra che in prevalenza ci si stia orientando in questa precisa direzione ${ }^{39}$.

Se proprio si volesse evocare la categoria, peraltro assai evanescente, degli "enti che svolgono funzioni di rilievo costituzionale" alla quale, per espressa previsione normativa, non si applica la disciplina in questione $\mathrm{e}^{40}$ - bisognerebbe, comunque, limitarne il raggio operativo alle attività inerenti alla finalità istituzionale di religione o di culto dell'ente ecclesiastico, le uniche atte propriamente a definirne il nucleo identitario più caratteristico. In altri termini, le garanzie assicurate dalla Carta fondamentale alle manifestazioni organizzative del diritto di libertà religiosa in forma collettiva, pur se particolarmente pregnanti e destinate per alcuni aspetti a operare anche fuori da quel nucleo, non possono

${ }^{39}$ Cfr. M. FERRANTE, Enti religiosi/ecclesiastici, cit., p. 178 ss.; V. MARANO, Sovranità della Chiesa ed enti ecclesiastici alla prova del diritto comune, in La Chiesa cattolica: la questione della sovranità, a cura di A. PEREGO, Vita e Pensiero, Milano, 2015, p. 85 ss.; D. FONDAROLI, A. ASTROLOGO, G. SILVESTRI, Responsabilità "amministrativa" ex d. lgs. n. 231 del 2001 ed enti ecclesiastici civilmente riconosciuti, in Stato, Chiese e pluralismo confessionale, cit., n. 38 del 2012, p. 1 ss.

40 Art. 1, terzo comma, del Decreto legislativo 8 giugno 2001, n. 231. Cfr. M.F. FERRERO, L'applicabilità del d.lgs. 231/2001 agli enti confessionali civilmente riconosciuti, in La responsabilità amministrativa delle società e degli enti, n. 2, 2014, p. 69 ss., il quale si richiama alla «necessità di garantire la continuità nello svolgimento delle "funzioni indefettibili negli equilibri costituzionali" da parte delle confessioni religiose (con il tramite dei propri enti)» (p. 76). 
autorizzare a ravvisare una funzione di rilievo costituzionale in qualsiasi attività "diversa" svolta dall'ente ecclesiastico ${ }^{41}$.

Né varrebbe porre l'accento sulla natura non profit dell'attività svolta, come pure si è talora fatto in passato ritenendo che destinatari naturali della disciplina fossero le società commerciali e che essa fosse «essenzialmente orientata a prevenire e sanzionare forme di criminalità 'di profitto', tipiche di soggetti che agiscono sul mercato» 42 , dal momento che sia il Codice del terzo settore (art. 30) sia la nuova normativa sull'impresa sociale (decreto legislativo 3 luglio 2017, n. 112) ormai esplicitamente rinviano alle disposizioni del decreto n. 231 del 2001, sia pure con una clausola di riserva che richiede una verifica di compatibilità da compiere di volta in volta 43 : per quel che qui interessa, qualunque sia la portata effettiva da riconoscere a quest'ultima riserva, il semplice richiamo del decreto n. 231 del 2001 vale, comunque, a escludere che la mancanza della finalità di lucro possa sempre e di per sé essere assunta come indice di incompatibilità tra lo statuto dell'ente e la normativa concernente la responsabilità da reato.

L'estensione della disciplina di cui al decreto n. 231 del 2001 all'ente ecclesiastico appare, dunque, limitatamente allo svolgimento delle attività diverse, inevitabile.

Le sanzioni interdittive, dovendo avere a oggetto la specifica attività cui si riferisce l'illecito dell'ente (art. 14, primo comma, decreto legislativo n. 231 del 2001) non potrebbero mai riguardare le attività inerenti alla finalità di religione o di culto.

Ma, soprattutto, l'adozione e l'efficace attuazione di un preciso modello organizzativo precostituito dal legislatore (i modelli di organizzazione e gestione, con i requisiti imposti dagli articoli 6 e 7 del decreto citato), che valgono (ove si fornisca la prova di particolari

41 È certamente vero che, già al livello costituzionale, le confessioni religiose godono di una tutela della loro autonomia più intensa di quella riconosciuta a partiti politici e sindacati (cfr. M.F. FERRERO, L'applicabilità, cit.), indicati nella Relazione ministeriale al decreto legislativo n. 231 del 2001 come figure di enti che svolgono funzioni di rilievo costituzionale; tuttavia, né i partiti né i sindacati hanno normalmente quella versatilità di azione che è, invece, tipica delle confessioni e dei relativi enti esponenziali.

42 V. MARANO, Sovranità della Chiesa, cit., p. 87.

43 Sia nell'art. 30, sesto comma, del Codice (decreto legislativo 3 luglio 2017, n. 117), sia nell'art. 10 del decreto legislativo n. 112 del 2017, è previsto che gli organi di controllo vigilano sull'osservanza della legge e dello statuto e sul rispetto dei principi di corretta amministrazione, anche con riferimento alle disposizioni del decreto legislativo 8 giugno 2001, n. 231, "qualora applicabili". 
circostanze a essi collegate) a esentare l'ente dalla responsabilità, a rigore, non sono obbligatorie, e, dunque, non possono essere ritenute necessariamente pregiudizievoli per l'autonomia organizzativa e strutturale dell'ente ecclesiastico.

Gli organi di amministrazione dell'ente, anziché adottare modelli etero-definiti particolarmente onerosi e di cui può essere dubbia la compatibilità con alcuni tipici elementi strutturali dell'ente ecclesiastico ${ }^{44}$, potrebbero decidere di fare affidamento sui propri modelli organizzativi e gestionali, specie se è ragionevole ritenere confinata a situazioni davvero eccezionali la commissione di fatti delittuosi ${ }^{45}$.

Fin qui sembrerebbe che l'applicazione del decreto n. 231 del 2001 all'ente ecclesiastico, con le precisazioni fatte, non incontri ostacoli insuperabili nella struttura di questo.

Va, tuttavia, considerato, per un verso, che la sanzione pecuniaria potrebbe intaccare il patrimonio dell'ente in misura tale da incidere anche sui fondi necessari per lo svolgimento dell'attività di religione o di culto, con conseguenze dirette, pertanto, sul perseguimento della finalità istituzionale; per altro verso, potrebbero rivelarsi un condizionamento indiretto molto forte all'adozione dei modelli di cui al decreto n. 231 citato quei provvedimenti regionali che subordinano alla presenza dei medesimi modelli la possibilità di intraprendere e mantenere rapporti con la pubblica amministrazione, secondo una scelta che ormai interessa pure "alcuni settori di attività (sanità e formazione) nei quali spesso operano anche gli enti ecclesiastici" 46 .

${ }^{44}$ M.F. FERRERO, L'applicabilità, cit., p. 79, secondo cui l'istituzione dell'organismo previsto dall'art. 6, primo comma, lett. $b$ ) del decreto legislativo n. 231 del 2001, dotato di autonomi poteri di iniziativa e di controllo e avente il compito di vigilare sul funzionamento e l'osservanza dei modelli e di curare il loro aggiornamento, potrebbe non essere compatibile con le caratteristiche strutturali di un ente ecclesiastico. Ricordo che negli enti di piccole dimensioni i compiti suddetti possono essere svolti direttamente dall'organo dirigente.

${ }^{45} \mathrm{Va}$, tuttavia, osservato che può non essere giustificata, con riguardo agli enti non profit in genere, una prognosi di rischio relativamente basso di commissione dei reatipresupposto di cui al decreto n. 231 del 2001, soprattutto in considerazione del fatto che tali enti possono entrare in rapporto con la pubblica amministrazione e ricevere agevolazioni o contributi pubblici: M. FERRANTE, Enti religiosi/ecclesiastici, cit., p. 177.

${ }^{46}$ V. MARANO, Sovranità della Chiesa, cit., p. 89, che richiama il decreto della Regione Lombardia n. 5808 dell'8 giugno 2010 e quello della Regione Lazio n. U00183 del 9 maggio 2013. 


\section{6 - (segue) b) la questione dell'assoggettabilità degli enti ecclesiastici alle procedure concorsuali}

In caso di svolgimento di vera e propria attività imprenditoriale, criticità ancora maggiori emergono per tutta una serie di profili concernenti l'estensione dello statuto dell'imprenditore.

$\mathrm{Si}$ pensi, anzitutto, alla questione riguardante la sottoposizione dell'ente alle procedure concorsuali. Anche in questo caso, bisogna fare i conti con il "duplice volto", per così dire, della clausola concordataria di estensione del diritto comune alle "attività diverse" nel rispetto della specialità dell'ente.

In vero, già da un punto di vista meramente pratico, si intuisce come non sia concepibile che un ente ecclesiastico imprenditore, avente una esposizione debitoria anche di diverse centinaia di milioni di euro, sia ritenuto immune rispetto a questo tipo di procedure, e venga lasciato operare sul mercato trascurando totalmente esigenze parimenti meritevoli di tutela giuridica, quali sono indubbiamente quelle dei creditori ${ }^{47}$.

Anche in questo caso, ne risentirà necessariamente l'autonomia gestionale e organizzativa dell'ente ove intervenga lo spossessamento dei beni e la conduzione dell'attività d'impresa da parte degli organi titolari della procedura in sostituzione di quelli dell'ente. Tuttavia, le ragioni che stanno a base della tutela dei creditori potrebbero giustificare una (temporanea) compressione dell'autonomia interna dell'ente, quale effetto di un ragionevole bilanciamento di contrapposte posizioni e avuto anche riguardo alla circostanza che il ricorso a queste procedure può risultare vantaggioso per lo stesso soggetto-imprenditore, interessato pertanto, in prima persona, a esservi ammesso ${ }^{48}$.

La giurisprudenza più recente, com'è noto, ha ritenuto che la clausola del rispetto della struttura e della finalità dell'ente risulti pienamente salvaguardata operandosi una scissione netta tra attività di carattere confessionale e attività imprenditoriale.

${ }^{47}$ Cfr. V. MARANO, Sovranità della Chiesa, cit., p. 95.

48 Rilevanti, in questo senso, sono anche alcune novità introdotte dal Codice della crisi d'impresa e dell'insolvenza (decreto legislativo 12 gennaio 2019, n. 14), che, limitando "gli spazi della liquidazione concorsuale e l'ingerenza delle autorità, prevedendo l'introduzione delle procedure che tendono a salvaguardare la continuità dell'impresa ed evitano la dispersione di valore del patrimonio", "sembrerebbe ridurre i potenziali elementi di incompatibilità tra il profilo soggettivo degli enti ecclesiastici e la normativa concorsuale": A. FUCCILLO, Diritto religioni culture, cit., p. 151. 
Si è infatti precisato che $\mathrm{l}^{\prime}$,'accertamento dello stato di insolvenza dell'ente e il suo assoggettamento alle procedure concorsuali non incidono [...] sulla struttura e sull'esistenza dell'ente" - che risulterebbero, dunque, pienamente rispettate -

"ma refluiscono direttamente solo sul segmento propriamente economico-imprenditoriale dell'attività dall'ente stesso esercitata; senza quindi comprimere la sua identità giuridica e in alcun modo impedirgli lo svolgimento delle attività di natura confessionale" ${ }^{\prime \prime 4}$.

Si tratta di un escamotage tutto sommato accettabile (a meno di accogliere la tesi che porta a differenziare la soluzione della questione della compatibilità con la struttura e finalità dell'ente a seconda delle caratteristiche della particolare procedura concorsuale in essere $)^{50}$, ma davvero poco convincente dal punto di vista della tecnica giuridica adottata per conseguirlo.

È singolare che, per rispettare la struttura e la finalità dell'ente ecclesiastico, il tribunale giunga a sezionarlo, creando sostanzialmente due distinti soggetti giuridici.

Dire che in questo modo si rispetta l'identità dell'ente è poco rispondente alla realtà delle cose, in quanto l'attività imprenditoriale, necessariamente secondaria rispetto alla finalità di religione o di culto, è pur sempre parte integrante, per così dire, sia della struttura (avendo riguardo ai caratteri che le ha impresso il diritto canonico) sia della stessa finalità dell'ente (almeno se rispetto a quest'ultima strumentale) ${ }^{51}$. L'effetto di una operazione di questo tipo è, in definitiva, quello di separare brutalmente le attività di culto dalle attività diverse, senza preoccuparsi di rispettare la configurazione originariamente impressa all'ente e da questo rivestita nell'ordinamento di provenienza.

49 Trib. civ. Roma, sez. fall., 30 maggio 2013, n. 432, in Quad. dir. pol. eccl., 2014/3, p. 956.

50 ... secondo la tesi di A.M. LEOZAPPA, Enti ecclesiastici e procedure concorsuali, in Stato, Chiese e pluralismo confessionale, cit., n. 4 del 2015, p. 22 ss.

51 Cfr. i condivisibili rilievi di A.M. LEOZAPPA, Enti ecclesiastici e procedure concorsuali, cit., p. 17 s. È evidente, del resto, che prevedere l'applicazione del diritto comune "nel rispetto della struttura e della finalità dell'ente" non può voler dire, banalmente, "lasciando che l'ente continui a svolgere attività di culto". Nel senso che "l'azione degli enti religiosi per la promozione dell'uomo [...] non può essere sezionata in religiosa e secolare", A.G. CHIZZONITI, La riforma del Terzo settore: aspetti di diritto ecclesiastico, in Enti religiosi e riforma del terzo settore, a cura di A. GIANFREDA e M. ABU SALEM, Libellula Edizioni, Tricase (Le), 2018, p. 17. 
Ancora più problematico appare il risultato della completa segregazione patrimoniale, cui giunge il tribunale di Roma, che porta a distinguere beni "per loro natura e destinazione" funzionali al compimento delle attività non imprenditoriali dell'ente (che restano fuori dalla procedura concorsuale) e beni costituenti "parte del patrimonio dell'imprenditore posto a garanzia generale delle obbligazioni da esso assunte" 52 , ancora una volta spiegabile solo alla luce delle ragioni pratiche richiamate all'inizio, ma non coerente con il principio inderogabile sancito dall'art. 2740 c.c. e con la normativa sull'amministrazione straordinaria nel caso concreto applicata ${ }^{53}$.

La menzionata disposizione del codice civile non potrebbe essere derogata neppure da una applicazione analogica del regime ora previsto per il ramo di Terzo settore dell'ente ecclesiastico, in relazione al quale, dopo le incertezze iniziali, si è introdotto un regime di perfetta segregazione dei beni destinati allo svolgimento delle attività di interesse generale, regime fondato, tuttavia, su un preciso atto di destinazione (costituzione del patrimonio destinato) ${ }^{54}$ inquadrabile, ad altri effetti, sotto la previsione dell'art. 19 della legge n. 222 del 1985. E se l'adozione di questo tipo di soluzione implica un qualche sacrificio per l'autonomia confessionale, va però ricordato che il legislatore propone l'adozione di uno schema organizzativo a fronte della fruizione di benefici promozionali. Per la disposizione in vigore, questa materia resta del tutto estranea rispetto a quelle esigenze di "rispetto della struttura e della finalità" dell'ente che sono purtuttavia ivi ribadite, ma solo in ordine al recepimento delle norme del Codice nel "regolamento" del ramo di attività 55 , "strumento fondamentale per definire l'area di specialità della

52 Trib. civ. Roma, 30 maggio 2013, cit., p. 957.

${ }_{53}$ Cfr., ancora, A.M. LEOZAPPA, Enti ecclesiastici e procedure concorsuali, cit., p. $16 \mathrm{s.}$

${ }^{54}$ L'art. 4, terzo comma, del decreto legislativo n. 117 del 2017, dopo le modifiche apportate dall'art. 66, primo comma, del d.-1. 31 maggio 2021, n. 77, convertito con modificazioni dalla legge 29 luglio 2021, n. 108, stabilisce che per le obbligazioni contratte in relazione alle attività svolte dal ramo Terzo settore, sia che riguardino quelle considerate di "interesse generale", sia quelle "diverse" (comprese, quindi, quelle commerciali), "gli enti religiosi civilmente riconosciuti rispondono nei limiti del patrimonio destinato. Gli altri creditori dell'ente religioso civilmente riconosciuto non possono far valere alcun diritto sul patrimonio destinato allo svolgimento delle attività" di cui sopra.

55 Cfr. la prima parte dell'art. 4, terzo comma, del decreto legislativo n. 117 del 2017, dove si ribadisce l'applicazione agli enti ecclesiastici delle norme del Codice a condizione che per le attività ivi considerate si adotti un regolamento che "ove non diversamente 
disciplina dei soggetti giuridici in questione" con riferimento al ramo Terzo settore 56 .

\section{7 - (segue) c) la questione delle divergenze risultanti dal Registro delle persone giuridiche e dal Registro delle imprese}

Un complesso intreccio tra componente strutturale dell'ente e normativa di natura civilistico-commerciale emerge anche per quanto riguarda le forme di pubblicità relative alle vicende interne e all'operatività dei controlli canonici relativamente all'attività imprenditoriale.

Sebbene la questione sia discussa dalla dottrina di diritto commerciale ${ }^{57}$, sin dalla Circolare del Ministero dell'industria, del commercio e dell'artigianato n. 3407/C del 9 gennaio 1997, per gli "organismi religiosi", in quanto svolgenti attività economiche che si collocano in una dimensione di sussidiarietà o di ausiliarità rispetto alla finalità istituzionale, si è prevista esclusivamente l'iscrizione nel Repertorio delle notizie economiche e amministrative (REA), tenuto dalle Camere di commercio, e non nel Registro delle imprese ${ }^{58}$.

previsto ed in ogni caso nel rispetto della struttura e della finalità di tali enti, recepisca le norme del [...] Codice e sia depositato nel Registro unico nazionale del terzo settore".

${ }^{56}$ I. BOLGIANI, Gli effetti della riforma, cit., p. 48.

57 Riferimenti in G. RUGOLO, L'ente ecclesiastico imprenditore: conflitto tra norme, poteri e giurisdizioni? Il caso della Fondazione Opera Diocesana Assistenza, in Vita notarile, n. 3, 2017, p. 1175.

58 In dottrina si è, pertanto, ribadito che "fino ad ora è pacifico che gli enti ecclesiastici non possano iscriversi al Registro delle imprese, nemmeno limitatamente alle attività d'impresa che gestiscono": così L. SIMONELLI, Il patrimonio destinato, in Enti religiosi e riforma del terzo settore, cit., p. 251. Cfr., pure, ID., Gli enti ecclesiastici e il repertorio economico amministrativo (REA), Supplemento a exLege, 2/2007, p. 5, secondo cui la Circolare citata implicitamente ribadisce che gli enti ecclesiastici "non devono iscriversi al Registro delle Imprese, in quanto il loro scopo principale ed essenziale non può mai essere la gestione di una attività economica organizzata". Reputano, invece, che "[n]ell'esercizio dell'attività d'impresa l'ente ecclesiastico [sarà] assoggettato allo statuto dell'imprenditore e anche al relativo regime di pubblicità di cui agli artt. 2188 e ss." c.c., A. FUCCILLO, R. SANTORO, L. DECIMO, Gli enti religiosi ETS. Tra diritto speciale e regole di mercato, Edizioni Scientifiche Italiane, Napoli, 2019, p. 89. Parla di iscrizione "facoltativa solo al ricorrere di attività di impresa", Trib. Catania, Ufficio del Giudice del Registro, decr. 19 aprile 2017, in Vita notarile, n. 3, 2017, p. 1171. Deve, comunque, essere iscritto alla apposita sezione del Registro delle Imprese il Ramo impresa sociale dell'ente religioso (art. 5, Decreto legislativo n. 112 del 2017). 
Ma anche qualora l'ente avesse provveduto all'iscrizione nel Registro delle imprese - che, a questo punto, alla luce della logica cui si ispira la predetta circolare, diventerebbe indice indiretto, ma inequivoco, dello svolgimento in via principale di attività commerciale - i problemi non mancherebbero, dal momento che, pure in questo caso, il Registro delle persone giuridiche, tenuto presso la prefettura, continua a essere la sede naturale, individuata dalle stesse fonti di derivazione concordataria (art. 5 e art. 18 legge n. 222 del 1985), dove dare pubblicità legale alle vicende riguardanti la costituzione dell'ente, il patrimonio, l'organizzazione interna con particolare riferimento ai nomi degli amministratori e rappresentanti, nonché le successive modificazioni.

In un caso verificatosi a Catania qualche anno fa, in cui l'Arcivescovo aveva rimosso, per gravi inadempienze, gli amministratori di una fondazione di culto dedita ad attività di assistenza, nominando un commissario straordinario, si è posto proprio un problema di divergenza tra le risultanze del Registro delle persone giuridiche, in cui il provvedimento vescovile era stato prontamente iscritto, e quelle del Registro delle imprese, nel quale il Conservatore, esercitando il proprio potere di controllo sulle condizioni richieste per l'iscrizione, l'aveva, invece, rifiutata, avendo ritenuto contrari allo statuto dell'ente i provvedimenti dell'Arcivescovo.

È molto interessante sottolineare come il Tribunale di Catania, cui si è rivolto l'amministratore dopo essersi visto respingere il reclamo dal Giudice del registro contro il rifiuto dell'iscrizione, abbia ritenuto pienamente efficaci le norme del codice di diritto canonico (can. 1279) che attribuiscono, anche nel silenzio sul punto dello statuto, al Vescovo un "generale potere di vigilanza" 59 in caso di negligenza del rappresentante legale, "in tal modo espressamente ammettendosi e confermandosi che la mancata previsione statutaria di un potere di revoca non esclude l'operatività del più generale potere di vigilanza previsto dal citato canone" 60 .

Così facendo, i giudici hanno, a mio avviso, correttamente valorizzato la speciale conformazione strutturale dell'ente, radicata nella normativa confessionale che ha, del tutto coerentemente, portato a rendere civilmente rilevante, quale "fonte immediata" di determinati poteri, il diritto canonico. L'iscrizione del provvedimento dovrebbe fugare eventuali "dubbi e incertezze nel terzo che opera con l'ente in ordine alla

59 Trib. Catania, ord. 14 giugno 2017, in Vita notarile, n. 3, 2017, p. 1185.

${ }^{60}$ Trib. Catania, ord. 14 giugno 2017, cit., p. 1188. 
sua legittima rappresentanza"61. Resta, tuttavia, da chiedersi cosa sarebbe accaduto qualora fosse rimasta la divergenza nelle risultanze dei due registri, i quali hanno finito con l'accogliere e mantenere per un certo tempo, con piena efficacia legale, l'indicazione contestuale di due diversi nomi di amministratori e rappresentanti ${ }^{62}$.

Anche in questa materia, a mio parere, non si può operare una suddivisione netta tra attività di religione e attività diverse: per la prima varrebbe quanto risultante dal Registro delle persone giuridiche, per la seconda quanto risultante dal Registro delle imprese.

Si pensi al regime dei controlli canonici, strettamente collegato, com'è noto, con le risultanze del primo registro, ma destinato a impattare anche sulle attività cui si riferisce il secondo. Pur riconoscendo l'importanza dei principi di certezza giuridica dell'attività negoziale che caratterizza l'attività d'impresa ${ }^{63}$, equivarrebbe, a mio parere, a trascurare totalmente la peculiarità strutturale dell'ente ecclesiastico, escludere l'operatività del sistema dei controlli canonici relativamente a quel tipo di attività.

Al contrario, l'operatività di quel sistema risulta ormai indirettamente confermata dal D.M. n. 106 del 2020, dove è previsto che il regolamento del ramo di attività di Terzo settore o di impresa sociale deve disciplinare, fra l'altro,

"i poteri di rappresentanza e di gestione, con specifica indicazione delle eventuali limitazioni e dei relativi controlli interni, se previsti

61 Si tratta di dubbi sollevati da P. CAVANA, Gli enti ecclesiastici nel processo di riforma, cit., p. 49 , nt. 118 , che mette ivi anche in evidenza il carattere "quanto mai vago e generico del concetto di negligenza" nel contesto del diritto canonico.

${ }^{62}$ Cfr., al riguardo, Trib. Catania, Ufficio del Giudice del Registro, decr. 19 aprile 2017, cit., p. 1171 s.: "[n]essun dubbio [...] che il contrasto tra le due forme di pubblicità (risultando iscritti quali legali rappresentanti soggetti differenti) possa generare confusione nei rapporti con i terzi, non potendosi attribuire efficacia prevalente ad una delle due iscrizioni: non è - però - compito di questo Giudice del Registro contemperare detta esigenza, ben potendosi fare ricorso al controllo giurisdizionale sulla legittimità dell'iscrizione presso il registro delle persone giuridiche" (fino alla scadenza del termine). "Né è possibile ritenere che iscritti gli atti presso il registro delle persone giuridiche la successiva iscrizione presso il registro delle imprese diventi una sorta di atto dovuto: nessuna norma prevede la prevalenza di un registro sull'altro. Né - ancora - è ipotizzabile una degradazione della iscrizione in questione a mera pubblicità notizia, non trovando tale tesi alcun conforto testuale e non avendo lo stesso istante qualificato $a b$ origine la sua richiesta in tal senso".

${ }^{63}$ Cfr. A. FUCCILlO, R. SANTORO, L. DECIMO, Gli enti religiosi ETS, cit., p. 88 s. 
dall'ordinamento confessionale, in conformità alle risultanze del Registro delle persone giuridiche" (art. 14, comma 2, lett. $g$ ),

nonché prevedere

"le condizioni di validità o di efficacia degli atti giuridici prescritte per gli enti religiosi civilmente riconosciuti dai relativi ordinamenti confessionali, ove tali condizioni abbiano rilevanza ai sensi di legge" (art. 14, comma 2, lett. $h$ ).

Con rischi, secondo qualche Autore, di una possibile responsabilità da culpa in vigilando a carico dell'autorità ecclesiastica in situazioni di dissesto economico dell'ente controllato ${ }^{64}$.

\section{8 - Il limitato carattere innovativo della locuzione "enti religiosi civilmente riconosciuti" presente nella normativa sul Terzo settore. Notazioni finali}

Una breve notazione vorrei, da ultimo, dedicarla alla locuzione "enti religiosi civilmente riconosciuti" che, come si sa, è stata, in seconda battuta, scelta dal legislatore per delimitare soggettivamente i destinatari della speciale disciplina di cui all'art. 4, terzo comma, del decreto legislativo n. 117 del 2017 (Codice del Terzo settore) e all'art. 1, terzo comma, del decreto legislativo n. 112 del 2017, riguardante l'Impresa sociale; una disciplina che, nel dettare le condizioni per l'applicabilità della riforma agli enti religiosi, non è direttamente intervenuta nei loro assetti organizzativi interni, per riguardo del peculiare collegamento strutturale con la confessione, lasciando che fossero gli enti stessi a recepire lo schema proposto dal legislatore nell'apposito "regolamento" e salvo sempre il "rispetto della struttura e della finalità" che li caratterizza.

Si potrebbe pensare che questa scelta, riproposta pure nei provvedimenti normativi di sostegno economico legati all'emergenza epidemiologica da Covid-1965, abbia segnato una sorta di "riposizionamento" del baricentro della disciplina relativa alle estrinsecazioni collettive della libertà religiosa, spostandolo dal nucleo almeno quantitativamente più rilevante, da sempre rappresentato dallo schema dell'ente "ecclesiastico" (o "confessionale"), verso il nuovo

\footnotetext{
${ }^{64}$ Questa tesi è sostenuta da P. CAVANA, Gli enti ecclesiastici nel processo di riforma, cit., p. 50.

${ }^{65} \mathrm{Cfr}$., per una analisi puntuale di questi provvedimenti in ordine al trattamento ivi riservato agli enti ecclesiastici, I. BOLGIANI, Gli effetti della riforma, cit., p. 77 ss.
} 
modello dell'ente "religioso"66. Si potrebbe, in questo senso, cogliere quell' ",apertura, non condizionata da pregiudizi, a una articolata e ricca pluralità di forme e di contenuti associativi, fondatizi e istituzionali" che aveva portato da tempo autorevole dottrina ad auspicare l'adozione proprio di un "cambio di nomenclatura" nella direzione accennata ${ }^{67}$.

Io sarei molto cauto.

Non so se il risultato sia quello di un "improprio impoverimento concettuale", come pure è stato sostenuto ${ }^{68}$, ma diversi elementi inducono a ritenere che il legislatore si sia mosso, in sostanza, nel solco della tradizione più consolidata, nonostante il termine adoperato ${ }^{69}$.

Anzitutto, basta riflettere sulla motivazione che ha indotto il legislatore a cambiare la soluzione adottata in prima battuta.

Si trattava di individuare una aggettivazione (la scelta avrebbe potuto tranquillamente cadere anche su quella di "confessionale") il più possibile comprensiva dal punto di vista delle confessioni destinatarie della garanzia, avendo dovuto dare una risposta alle preoccupazioni espresse dal Consiglio di Stato ${ }^{70}$ in ordine alla formulazione precedentemente adottata, da quel preciso punto di vista (dei destinatari confessionali) troppo selettiva e, quindi, di dubbia compatibilità col principio di laicità dello Stato $(\mathrm{o}$, ancor prima, di eguale libertà di tutte le confessioni religiose).

In secondo luogo, che si abbia a che fare con un ente confessionale, collegato con uno specifico gruppo religioso, risulta dalla stessa clausola di salvaguardia del rispetto della struttura dell'ente, richiamata nell'art. 4, terzo comma, del Codice (e nella corrispondente previsione del decreto $\mathrm{n}$. 112 cit.), che evidentemente evoca quello specifico collegamento ${ }^{71}$; in

${ }^{66}$ Ha sottolineato l'avvenuto "spostamento dell'asse normativo del diritto unilaterale di produzione statale dal polo dell'ecclesiasticità a quello della religiosità degli enti, entrambi garantiti e protetti dall'art. 20 Cost.", M. FERRANTE, Enti religiosi/ecclesiastici, cit., p. 61.

${ }^{67}$ Cfr. S. BERLINGÒ, Enti e beni religiosi in Italia, cit., p. $67 \mathrm{~s}$.

${ }^{68}$ P. CONSORTI, L'impatto del nuovo Codice, cit., p. 8.

${ }^{69}$ Invita, invece, a non sottovalutare la novità terminologica, M. FERRANTE, Enti religiosi/ecclesiastici, cit., p. 48, che arriva a parlare, per effetto della scelta del legislatore, di "un'obsolescenza sopravvenuta ma non programmata della normativa di derivazione concordataria e pattizia con la conseguente progressiva perdita di senso giuridico della nozione di ecclesiasticità applicata agli enti che sembra avere ormai un carattere residuale, essendosi ormai quasi del tutto svuotata di contenuto" (ivi, p. 57).

70 Cons. St., commissione spec., parere 14 giugno 2017, n. 1405.

71 Cfr. A. GIANFREDA, Autonomia confessionale e sistema delle fonti del diritto ecclesiastico. Riforma del terzo settore e tutela della privacy: un banco di prova per la produzione 
termini ancora più espliciti si è espresso poi il D.M. n. 106 del 2020, il quale, in modo inequivoco e a più riprese, presuppone in definitiva, come è stato osservato, che "anche gli enti religiosi civilmente riconosciuti devono essere riconducibili a una confessione"72.

Ulteriori perplessità suscita la tesi secondo la quale la possibilità, prevista dall'art. 22 del Codice, di acquistare la personalità giuridica mediante l'iscrizione nel Registro del terzo settore possa valere anche per $l^{\prime}$ ente ecclesiastico privo di riconoscimento ${ }^{73}$.

A parte altre considerazioni, mi sembra dirimente la circostanza che lo stesso art. 22 precisi che tale possibilità è prevista "in deroga al decreto del Presidente della Repubblica 10 febbraio 2000, n. 361" e non alle norme applicabili agli enti ecclesiastici, peraltro garantite, come si sa, da specifica copertura costituzionale.

Concludo osservando che la specialità del regime giuridico dell'ente ecclesiastico, abbandonata ogni antica pretesa di privilegio, più o meno motivata e più o meno estesa, dovrebbe servire a garantire a esso la possibilità di operare nell'ordinamento dello Stato conservando i tratti più caratteristici della propria identità confessionale al fine di assicurarne anche la relativa "visibilità" e "rappresentatività" esteriore.

Il che può pure risultare compatibile con la presenza attiva nel mercato - accanto alle forme organizzative assistenziali (religiosamente ispirate) operanti secondo gli schemi puramente civilistici - purché in esso l'apporto degli enti delle confessioni religiose si caratterizzi per una certa infungibilità dei beni o dei servizi offerti (per qualche aspetto, dunque, qualitativamente irriducibili a quelli assicurati da un qualsiasi soggetto

normativa confessionale, in Costituzione, religione e cambiamenti nel diritto e nella società, a cura di P. CONSORTI, Pisa University Press, Pisa, 2019, p. 353, secondo cui resta essenziale $1^{\prime}$ «elemento della c.d. "ecclesiasticità strutturale". Gli enti, senza legame strutturale di carattere confessionale, ma con una religiosità solo funzionale [...], potrebbero accedere alla normativa del Terzo settore, come enti del Terzo settore tout court, orientati religiosamente, "di tendenza" per così dire, senza passare per la costruzione di un "ramo" di attività del Terzo settore».

72 Per questo condivisibile rilievo, si veda P. LO IACONO, Gli enti ecclesiastici, cit., p. 70. Sui dubbi relativi a un possibile contrasto con l'art. 20 Cost. della normativa in esame per il trattamento riservato agli enti religiosi privi di riconoscimento (in relazione a quello riservato agli enti di diritto comune, anch'essi non riconosciuti), cfr. P. FLORIS, Enti religiosi e riforma del Terzo settore, cit., p. 16.

${ }^{73}$ In questo senso, ad esempio, M. FERRANTE, Enti religiosi/ecclesiastici, cit., p. 57. 
privato) 74 , senza scendere a discutibili forme di "scambio" o "compromesso" nella testimonianza dei valori propriamente religiosi ${ }^{75}$.

${ }^{74}$ Diversi Autori pongono l'accento su tale aspetto. Cfr., pur tutti, A. MANTINEO, Il Codice del terzo settore: punto di arrivo o di partenza per la palingenesi degli enti religiosi?, in Stato, Chiese e pluralismo confessionale, cit., n. 27 del 2018, p. 5 s., il quale si chiede se il Codice del terzo settore non "rappresenti una prospettiva entro cui gli enti religiosi sono chiamati, più di ieri, ad armonizzarsi con altri organismi ed enti per meglio promuovere l'uomo e i suoi diritti civili e sociali, senza smarrire o stemperare la loro natura particolare, nel rispetto delle loro motivazioni e finalità" e M. FERRANTE, Enti religiosi/ecclesiastici, cit., p. 72.

75 È vero che l'azione della Chiesa nella realtà temporale, se intesa come "azione di testimonianza di valori" è a "rischio di marginalizzazione"; ma non è meno incombente il rischio, parimenti segnalato, "di omologazione", se intesa come "mera attività di assistenza tout court": P. CAVANA, Gli enti ecclesiastici nel processo di riforma, cit., p. 31. A conti fatti, è meglio non trovarsi a dovere barattare, come è stato scritto, la "rinuncia alla formale riconoscibilità" della specifica identità confessionale con "la fruizione di una normativa (evidentemente) non pensata per il rispetto e lo sviluppo delle finalità ed autonomie religiose": A. FERRARI, Le linee generali della Proposta di legge sulla libertà di coscienza e di religione, in La legge che non c'è. Proposta per una legge sulla libertà religiosa in Italia, a cura di R. ZACCARIA, S. DOMIANello, A. FerRARI, P. Floris, R. MAZZOlA, il Mulino, Bologna, 2019, p. 78. 